\title{
En búsqueda de un sistema de formación de habilidades en matemáticas
}

\section{In search of a mathematics skills training system}

\author{
Nelson Claudio Córdova Rosas \\ Universidad Santa María, Ecuador
}

Autor para correspondencia: ncordova@usm.edu.ec

Fecha de recepción: 6 de Diciembre de 2016 - Fecha de aceptación: 10 de Enero de 2017

Resumen: Este documento presenta un sistema de aprendizaje metodológico que busca desarrollar habilidades de pensamiento matemático, fortaleciendo el trabajo mental. Este sistema consta de tres etapas: la conceptualización semántica, la aplicación práctica y el desarrollo crítico. Estas etapas permiten el aprendizaje de manera concisa a través de la internalización de habilidades. Paralelamente se van logrando etapas de construcción del conocimiento; partiendo desde lo más simple hacia lo más complejo. Con esto se pretende contribuir al proceso enseñanza y aprendizaje de la matemática tanto para estudiantes como profesores; de tal manera que éstos puedan desarrollar habilidades lógicas de pensamiento estructurado en el área de las matemáticas. Este sistema se basa en la investigación de Córdova - Estay (2002), dónde se presenta un sistema escalonado para la congruencia educativa, para el aprendizaje de las Matemáticas. Éste promueve la internalización paulatina de habilidades en cadenas, que van en asenso por etapas de consolidación. El sistema que se propone en el presente artículo, es un apoyo y complemento para el sistema de aprendizaje de las matemáticas.

Palabras clave: Congruencia Educativa Matemática; Aprendizaje Continuo; Sistema de aprendizaje y enseñanza de Matemáticas

\begin{abstract}
This document presents a system of methodological learning that seeks to develop mathematical thinking skills, strengthening our mental capacities. This system consists of three stages: synthetic conceptualization, practical application and critical development. These stages enable learning in a concise way through the internalization of skills. At the same time, we go through stages of knowledge construction; starting from simple to the most complex aspects. This is intended to contribute to the teaching and learning process of mathematics for both students and teachers; so that they develop logical thinking skills structured in the area of mathematics. This system is based on the research of Córdova - Estay (2002), which presents a stepped model of educational congruence for the learning of Mathematics. It promotes the gradual internalization of chain-linking skills, which are supported by a series of stages of consolidation. The system proposed in this article is a support and complement for this learning model.
\end{abstract}

Key words: Mathematical Educational Congruence; Continuous Learning; Mathematics learning and teaching system. 


\section{Introducción}

Hace ya algunas décadas y por muchas generaciones en América Latina se vive con recelo por la Matemática, mensaje muchas veces subliminal y otras veces directo trasmitido por las generaciones a generaciones, producto de un sistema escolar plagado de inconsistencias y débil preparación de los maestros. Sistema que se enajena de considerar principios importantes que intervienen en el proceso de enseñanza y aprendizaje, tales como: diferencias individuales, velocidad adecuada de la enseñanza, metodología del aprendizaje, dificultad de los temas, desarrollo de habilidades, proyección futura de los temas, etc. Pero, ¿cuál es el problema real en la preparación que presentan los bachilleres que se reciben en las universidades?

Durante muchos años, en observaciones y evaluaciones efectuadas en preuniversitarios en Chile y Ecuador, se ha podido constatar una deficiente preparación de los estudiantes en términos conceptuales y en el conocimiento de la lógica de los procedimientos en matemáticas. En estudios realizados en nuestra Universidad la mayoría de estos alumnos presentan confusión y olvido de la mayor parte de los procedimientos elementales y fórmulas fundamentales, además carecen de una preparación en habilidades que involucran análisis y síntesis que agrava aún más la situación.

Por estas razones es que han proliferado cursos propedéuticos fuera y al interior de las universidades, los cuales pretenden proporcionar una nivelación y que en la mayoría de los casos ha sido insatisfactoria debido principalmente a que las falencias en los estudiantes son demasiado grandes y no es posible construir el conocimiento en tan poco tiempo. Por su parte en los primeros años de las carreras de ingeniería se ha tornado ineludible desarrollar compendios básicos de Matemática. Esto nos conduce a pensar que las universidades están conscientes de esta realidad y que por varios años han tenido que asumir un papel formativo que debía estar resuelto desde el colegio. Sin embargo, aún con este esfuerzo, un gran porcentaje de casos no alcanza a completar una formación básica necesaria.

En cursos de Matemática en universidades de prestigio principalmente en carreras de Ingeniería se observa un común denominador en la impartición de las asignaturas de esta naturaleza: el tiempo limitado. El escaso tiempo que se le dedica a la enseñanza de las Matemáticas imposibilita a los estudiantes desarrollar completamente las habilidades que necesitan para enfrentar el reto universitario.

En este artículo se propone una estrategia general que pretende conjugar paralelamente, en cada aprendizaje, un conjunto de habilidades generales con competencias. Su objetivo principal es la formación del pensamiento matemático donde estas habilidades juegan el papel principal.

Para exponer la estrategia, utilizamos un ejemplo clásico, que exhibe como organizar el aprendizaje utilizando diversos elementos matemáticos que permitirán formar una base metodológica en el estudiante.

\section{Elementos Imprescindibles Para Considerar En Un Sistema De Aprendizaje.}


En este artículo nos enmarcamos dentro del enfoque histórico-cultural de Vygotsky (1979) el cual señala que todo aprendizaje utiliza experiencias y aprendizajes previos que de alguna manera interactúan entre sí con los nuevos conocimientos. Establece el Nivel evolutivo real, el cual se refiere a lo que el aprendiz puede realizar por si solo y el Nivel de desarrollo potencial, que se refiere a lo que el aprendiz puede realizar con una ayuda externa. (Cabrera \& Mazzarella, 2001)

Esta base histórico-cultural, es para nuestro parecer, crítica, para establecer un buen aprendizaje, especialmente en la Matemática Universitaria. Sin embargo, existen una serie de otras variables a considerar que afectan directamente al aprendizaje, Pero:

¿Qué elementos mínimos, son supuestamente aquellos que se deben desarrollar?

Podrían listarse una infinidad de ellos, sin embargo nombraremos aquellos más manejables y que estén, según nuestro criterio, considerados dentro de los elementos mínimos de trabajo; a saber:

- Conocimientos básicos del Área

- Habilidades básicas

- La conceptualización de los elementos primarios

- La lógica de los procedimientos

- Niveles de aplicación de los temas

- Herramientas de apoyo

Junto con conocimientos básicos lo más aproximado al ideal que la universidad requiere es quizás, estudiantes que tengan desarrolladas, o en vías de desarrollo, un número importante de las habilidades matemáticas básicas. Sin embargo según Flores (2001) citado por (Santaolalla, 2009) manifiesta que el aprendizaje de conceptos se aborda de manera estructural, pues, son estructuras mentales las que se deben modificar y mejorar en concordancia con lo propuesto por Vygotsky, el cual menciona que las funciones en el desarrollo intelectual aparecen en dos momentos, primero, en una fase social y luego en una fase individual, es decir, interpsicológicamente e intrapsicologicamente Vygotsky (1979). Además también es necesario considerar cualidades fundamentales del aprendizaje matemático en la concepción actual.

1. El aprendizaje matemático se realiza a través de experiencias concretas.

2. El aprendizaje tiene que arrancar de una situación significativa para los alumnos.

3. La forma en que los aprendices pueden llegar a incorporar el concepto a su estructura mental es mediante un proceso de abstracción que requiere de modelos.

4. Una de las formas de conseguir que el aprendizaje sea significativo para los alumnos es mediante el aprendizaje por descubrimiento.

5. No hay un único estilo de aprendizaje matemático para todos los alumnos. (Santaolalla, 2009)

Sin embargo es necesario tener cierta base metodológica sustentada en dos elementos vitales para el desarrollo de la matemática: las Habilidades Matemáticas y las Competencias Matemáticas básicas, donde una combinación intencionada de ellas en el aprendizaje es una alternativa que potencia la actuación del estudiante y crea necesariamente escenarios más 
prometedores para el crecimiento matemático de los jóvenes y para el desarrollo de la enseñanza para los maestros.

A nuestro entender una habilidad matemática: es una exacta mezcla de capacidades, operaciones sistematizadas y conductas, orientadas a lograr un objetivo matemático específico. Y por otra parte entendemos por competencia matemática "Una óptima amalgama de Conocimientos, habilidades, aptitudes y valores que dotan al ser humano para tener un excelente desempeño en una actividad específica" (Córdova \& Oliveros, 2014). Entendemos también que una competencia conlleva habilidades, por lo tanto vamos a considerar que las habilidades estarán enmarcadas dentro de una competencia.

\section{Habilidades Y Competencias Básicas}

Las habilidades básicas propuestas por H. Hernández y ampliadas por Juan delgado, son las siguientes (Delgado, 1999)

Definir, identificar, interpretar, calcular, graficar, recodificar, algoritmizar, demostrar, representar, comparar, controlar, fundamentar, modelar.

Creemos que es posible establecer la formación siguiendo un orden secuencial en cada aprendizaje para el desarrollo del pensamiento creando una amalgama con estas habilidades.

\section{Tabla 2. Habilidades Matemáticas}

Definir: Es establecer mediante una proposición las características necesarias y suficientes del objeto de estudio.

Identificar: Es distinguir el objeto de estudio matemático sobre la base de sus rasgos esenciales. Es determinar si el objeto pertenece a una determinada clase de objetos que presentan ciertas características distintivas

Interpretar: Es atribuir significado a las expresiones matemáticas de modo que están adquieran sentido en función del propio objeto matemático o en función del fenómeno o problema real que se aborde.

Calcular: Es la forma esencial de un algoritmo que puede llevarse a cabo de forma manual, verbal, (oral o escrita), mental y mediante el uso de tablas, calculadoras $\mathrm{u}$ ordenadores.

Representar: Hacer presente una cosa en forma escrita por medio de signos, imágenes o símbolos.

Comparar: Es establecer una relación entre lo cualitativo o cuantitativo que hay entre dos entes matemáticos de un mismo conjunto o clase.

Graficar: Representar datos numéricos por medio de coordenadas o dibujos que hacen visible la relación o gradación que esos datos guardan entre sí.

Recodificar: Es transferir la denominación de un mismo objeto de un lenguaje a otro. Es expresar el mismo tipo de objetos a través de formas diferentes, no es más que la utilización de signos diferentes para un mismo modelo.

Algoritmizar: Es plantear una sucesión escrita de operaciones matemáticas que describan un procedimiento conducente a la solución de un ejercicio o problema.

Controlar: Comprobación, inspección, fiscalización o intervención en el desarrollo de situaciones matemáticas.

Fundamentar: Establecer principios, reglas, teoremas que validan una acción o propuesta.

Demostrar: Es establecer una sucesión finita de pasos para fundamentar la veracidad de una proposición o su refutación.

Modelar: Es asociar a un objeto no matemático un objeto matemático que represente determinados comportamientos, relaciones o características suyos.

Fuente: (Delgado, 1999) 
Además consideramos como marco de referencia ir considerando 5 niveles formados por grupo de competencias matemáticas esenciales. Esta idea traducida y simplificada en términos de competencias: se presenta de la siguiente manera en (Córdova \& Oliveros, 2014)

\section{Competencias Matemáticas básicas según PISA.}

\begin{tabular}{lll}
\hline 1. & Comunicar & 5. Realizar cálculos operativos \\
\hline 2. & Representar y simbolizar & 6. Pensar y razonar \\
\hline 3. & Utilizar lenguaje y operaciones simbólicas & 7. Argumentar \\
\hline 4. & Utilizar ayudas y herramientas & 8. Modelizar \\
\hline & & 9. Plantear y resolver problemas. \\
\hline
\end{tabular}

Fuente: (Córdova, Oliveros, 2014)

Córdova-Oliveros (2014) presentan una alternativa para organizar el aprendizaje de las competencias básicas matemáticas, es decir considerando un orden lógico de desarrollo como un sistema circular que a la larga promueve escenarios que pueden inspirar a la innovación y creatividad. (Córdova, Oliveros, 2014).

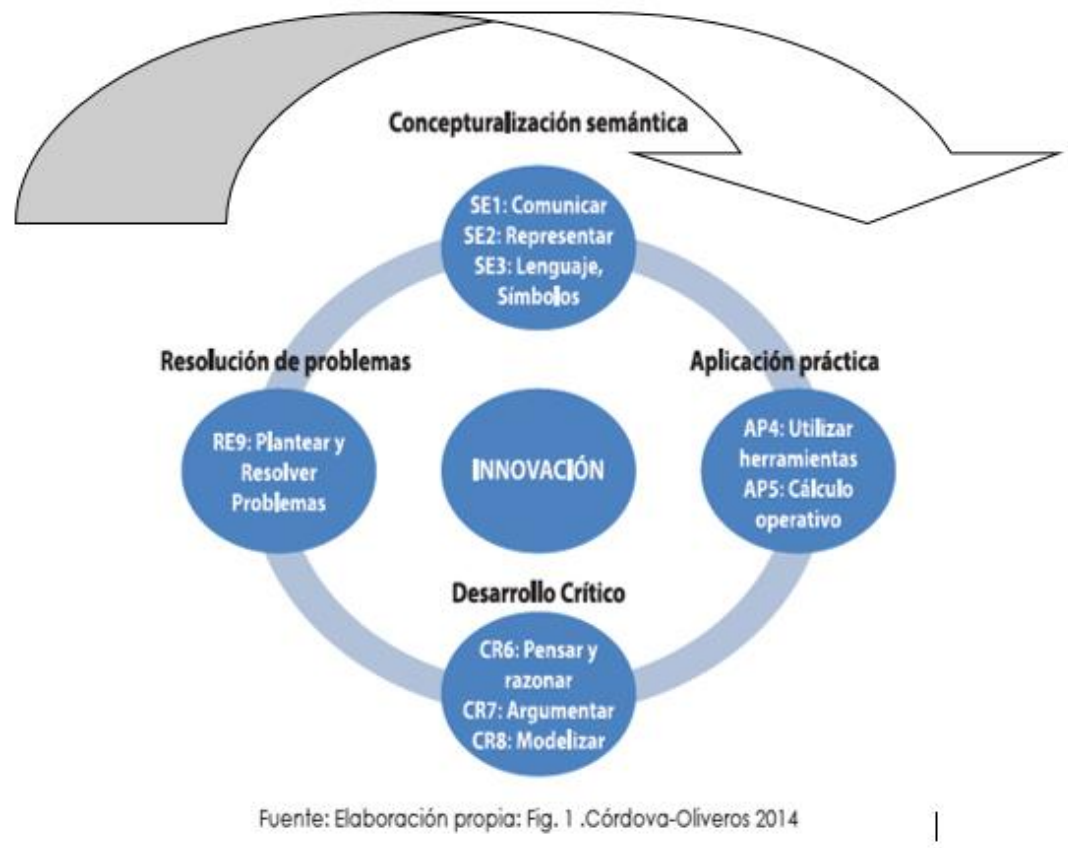

Partiendo de una Conceptualización semántica a través de la ayuda de competencias tales como: Comunicación, Representación, Manejo del lenguaje, podamos dar sentido a los elementos matemáticos con los cuales vamos a trabajar, pero esta primera etapa no estaría completa si no le damos una Aplicación práctica y aterrizada que permita dos cosas; darle vida a los conceptos y visualizar su aplicación utilizando las herramientas aprendidas. Si la primera fase se concreta satisfactoriamente, entonces podemos avanzar a la segunda, considerada superior, por la amalgama de habilidades y razonamientos involucrados en el Desarrollo Crítico cuyo desarrollo se sustenta de competencias tales como: Pensar y razonar, Argumentar y Modelizar en esta nueva fase es donde es posible introducir y preparar al estudiante para la siguiente etapa crucial que es Plantear y Resolver problemas, etapa que algunos la tienen como 
objetivo final y está muy bien porque es una etapa superior y muy difícil superar con éxito. Si bien es sabido que los estudiantes tienen mucha dificultad en resolver problemas, Sin embargo creemos que para completar la formación a un alto nivel, se necesita acceder a una última etapa y final que es: La Creatividad y la Innovación en la cual podemos contribuir a desplegar la imaginación de los maestros y de los estudiantes.

Esta concepción de crecimiento paulatino en el manejo de habilidades, es una idea presentada por el investigador Ramírez et al. (1988) La cual fue aplicada en la Universidad Santa María Campus Guayaquil. (Ramírez P., Recabaren, \& Palma, 1988).

\section{Aplicación Del Modelo De Ramírez}

En una experiencia con la aplicación del modelo de Ramírez, en la Universidad Santa María Campus Guayaquil entre los años 1998-2005 se pudo "demostrar" que el desarrollar esta forma de interpretar el aprendizaje, logró una mejora de los resultados de los alumnos en las materias de Matemáticas para ingeniería. (Córdova \& Estay-Niculcar, 2002).

Tabla 1. Porcentajes de aprobación anual en asignaturas de matemáticas. Universidad Santa María Campus Guayaquil.

\begin{tabular}{|c|c|c|c|c|c|c|c|c|c|c|}
\hline $\begin{array}{l}\text { PROMEDIOS ANUALES DE } \\
\text { APROBACION }\end{array}$ & 1996 & 1997 & 1998 & 1999 & 2000 & 2001 & 2002 & 2003 & 2004 & 2005 \\
\hline Int. al Álgebra & & $57 \%$ & $61 \%$ & $52 \%$ & $65 \%$ & $82 \%$ & $85 \%$ & $70 \%$ & $45 \%$ & $68 \%$ \\
\hline Int. al Cálculo & & $47 \%$ & $63 \%$ & $58 \%$ & $68 \%$ & $61 \%$ & $68 \%$ & $51 \%$ & $53 \%$ & $65 \%$ \\
\hline Álgebra I & $25 \%$ & $39 \%$ & $32 \%$ & $51 \%$ & $55 \%$ & $82 \%$ & $58 \%$ & $61 \%$ & $58 \%$ & $66 \%$ \\
\hline Cálculo I & $16 \%$ & $43 \%$ & $43 \%$ & $52 \%$ & $78 \%$ & $82 \%$ & $68 \%$ & $81 \%$ & $49 \%$ & $51 \%$ \\
\hline Cálculo II & & $52 \%$ & $55 \%$ & $70 \%$ & $60 \%$ & $46 \%$ & $80 \%$ & $83 \%$ & $62 \%$ & $68 \%$ \\
\hline Álgebra II & & $65 \%$ & $38 \%$ & $72 \%$ & $83 \%$ & $50 \%$ & $73 \%$ & $81 \%$ & $70 \%$ & $49 \%$ \\
\hline Cálculo III & & & $39 \%$ & $62 \%$ & $42 \%$ & $73 \%$ & $83 \%$ & $74 \%$ & $72 \%$ & $67 \%$ \\
\hline Promedio de aprobación por año & $21 \%$ & $48 \%$ & $49 \%$ & $59 \%$ & $62 \%$ & $68 \%$ & $75 \%$ & $73 \%$ & $58 \%$ & $62 \%$ \\
\hline
\end{tabular}

Fuente: Elaboración propia (Córdova \& Estay-Niculcar, 2002).

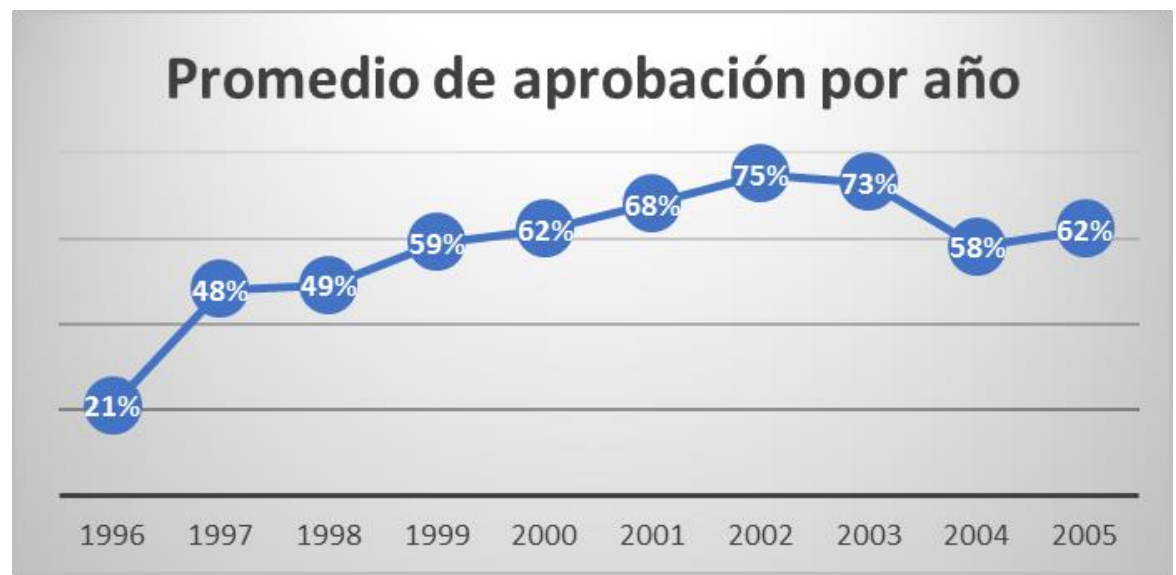

Fuente: Elaboración propia (Córdova \& Estay-Niculcar, 2002).

El investigador Ramírez et al. (1988) presentó un "modelo" que establece niveles de madurez en una secuencia lógica para el aprendizaje, esta consta de 4 niveles que son etapas que se presentan generalmente en aprendizajes efectivos en el área de matemáticas. A saber: 
.Reproductivo, Transferencial, Crítico y Creativo. En la antigüedad los Gestalistas los habían definido en dos; Reproductivo y Productivo, en este caso el Productivo sería equivalente a los tres últimos de Ramírez (Ramírez P., Recabaren, \& Palma, 1988).

Esta experiencia nos ha motivado a desarrollar la idea, de que es posible definir un sistema de aprendizaje, que paulatinamente vaya desarrollando en los estudiantes las habilidades más relevantes para el estudio de las Matemáticas universitarias. Al mismo tiempo, ir avanzando en el sistema de competencias matemáticas básicas definidas por el proyecto PISA de la OCDE como punto de partida.

La idea central de este sistema es que en la medida que se va impartiendo el contenido y fijando conceptos, en forma paralela se vaya desarrollando un conjunto acotado de habilidades matemáticas, utilizando la vía pedagógica más prudente. Estas habilidades deben estar en relacionadas o contenidas dentro de las competencias a considerar y que se pueden agrupar como lo indica la figura 1. (Córdova, Oliveros, 2014).

\section{Sistema Básico De Formación De Habilidades}

Un sistema de formación de habilidades consta de tres fases o etapas Entrada-ProcesoSalida las cuales interactúan desarrollando cada una un objetivo particular que a su vez ayuda a concretar un objetivo general, dentro el proceso completo de una unidad de aprendizaje.

En primer lugar se fija el objetivo general que puede ir desde el desarrollo de una competencia o contemplar desarrollo de habilidades en un tema específico. Por ejemplo: "Resolver problemas matemáticos mediante diferentes estrategias, procedimientos y recursos, desde la intuición hasta los algoritmos". O conseguir el desarrollo de un resultado de aprendizaje ligado a una competencia, esto dentro de un marco pedagógico constituido por tres elementos necesarios. Contenido, Metodología y Evaluación. (Rico, 1997).

La Primera Etapa, Entrada: la denominamos Conceptualización Semántica. Es aquella que proporciona las bases del nuevo aprendizaje con herramientas, habilidades específicas, procesos, etc., pero lo fundamental es la comprensión misma de todos los elementos previos considerados imprescindibles para el desarrollo efectivo de la nueva unidad. En este nivel también se necesita la comprensión de nuevos conceptos en un nivel práctico, porque esta etapa está concebida para consolidar esa comprensión, de manera que cada elemento matemático pueda ser interpretado y estudiado desde varios puntos de vista; por ejemplo, se necesita para la formación de cada concepto: 1 . Sistema de conocimientos previos 2.manejo de elementos de interpretación visual o gráfica, 3. Notaciones matemáticas, 4. Aplicaciones dentro de la matemática misma, 5. Ayudas didácticas que aumenten la percepción del objeto matemático. 6 . Herramientas de apoyo.

La Segunda Etapa, Proceso: la denominamos Aplicación Práctica y es donde el nuevo concepto encuentra su real desarrollo en su utilización para resolver situaciones pragmáticas. En este punto el estudiante se necesita 1. Una metodología 2. Una ejercitación estructurada, 3. Problemas aplicados, 4.Tiempo para desarrollar el trabajo, 5. Una asesoría constante. 
La Tercera Etapa, Salida: la denominamos Desarrollo Crítico, etapa de consolidación y despliegue de las habilidades, de conceptos aprehendidos en las etapas anteriores y en la que se debe exponer el cumplimiento del objetivo general. Si esto no es efectivo, entonces el estudiante debe volver a las etapas iniciales a modo de realimentación del proceso y revisar Metacognitivamente en que instante estuvo la falla o si se lograron los objetivos de aprendizaje (En este caso: desarrollar el nivel crítico en forma efectiva), porque también es necesario revisar retrospectivamente lo que se hizo bien y revisar cuales fueron las etapas o momentos claves del aprendizaje. Esto es con el objeto de afianzar el conocimiento actual y fortalecer conocimientos previos del aprendizaje posterior.

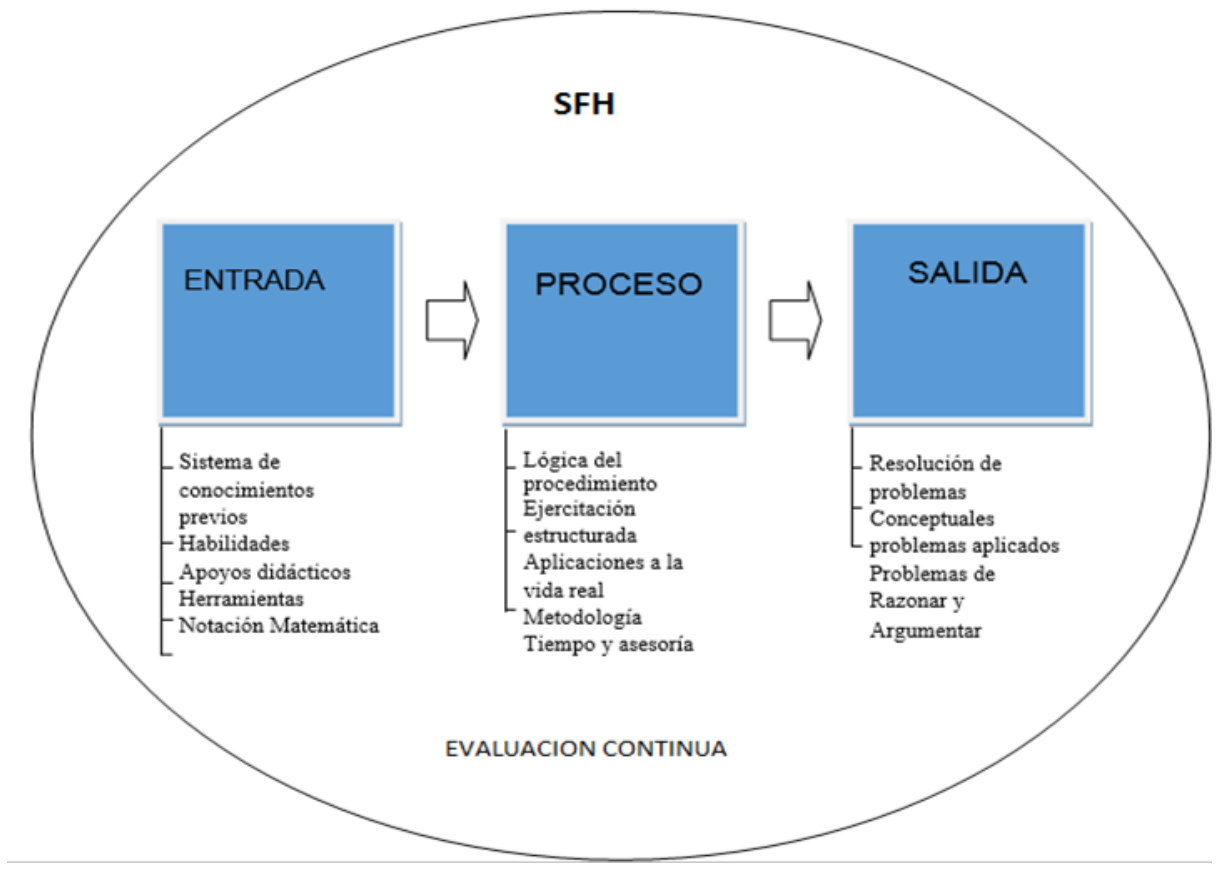

Fig. 2. Sistema básico de formación de habilidades Fuente: Elaboración propia.

\section{Ejemplo De Sistema Básico De Formación De Habilidades}

Un tema interesante y muy importante es Límites de funciones, materia que se imparte en los primeros cursos de Cálculo y que por lo general ha sido un dolor de cabeza para los estudiantes de primer año en carreras de ciencias duras, tales como las ingenierías y Licenciaturas en Ciencias. Aquí se muestra un ejemplo de cuáles son los elementos mínimos a considerar en las tres etapas de este sistema de formación de habilidades para el tema antes mencionado.

\section{Tema: límites de funciones racionales}

Objetivo General: El Alumno Será Capaz De Analizar, Interpretar, Y Resolver Todos Los Tipos De Límites De Funciones Racionales.

$\begin{array}{llll}\text { PASOS Sub-sistemas } & \text { Componentes } & \text { Habilidades } & \text { Evaluación }\end{array}$




\begin{tabular}{|c|c|c|c|c|}
\hline \multirow[t]{5}{*}{$\begin{array}{l}1 \\
\text { Conceptualización } \\
\text { Semántica } \\
\text { (entrada) }\end{array}$} & $\begin{array}{l}\text { Sistema de } \\
\text { conocimientos } \\
\text { previos }\end{array}$ & $\begin{array}{l}\text { Conceptos de } \\
\text { función, imagen, } \\
\text { pre imagen y gráfica } \\
\text { Concepto de } \\
\text { aproximación } \\
\text { Conceptos } \\
\text { aritméticos de los } \\
\text { números } \\
\text { Factorización de } \\
\text { polinomios, } \\
\text { racionalización }\end{array}$ & $\begin{array}{l}\text { Interpretar } \\
\text { Identificar } \\
\text { Representar }\end{array}$ & $\begin{array}{l}\text { Evaluación } \\
\text { diagnóstica: establece } \\
\text { por cada estudiante } \\
\text { 1.niveles de } \\
\text { habilidades adquiridas } \\
\text { y requeridas. } \\
\text { 2. operaciones básicas } \\
\text { requeridas } \\
\text { 3.- nivel de escritura }\end{array}$ \\
\hline & $\begin{array}{l}\text { Concepto grafico } \\
\text { del límite }\end{array}$ & $\begin{array}{l}\text { Percepción visual: } \\
\text { Representación } \\
\text { gráfica } \\
\text { Interpretación } \\
\text { semántica }\end{array}$ & $\begin{array}{l}\text { Interpretar } \\
\text { Representar } \\
\text { Graficar } \\
\text { Identificar }\end{array}$ & $\begin{array}{l}\text { Evaluación } \\
\text { permanente de avance }\end{array}$ \\
\hline & $\begin{array}{l}\text { Representación } \\
\text { matemática }\end{array}$ & $\begin{array}{l}\text { Semántica y } \\
\text { lenguaje } \\
\text { Denotación }\end{array}$ & $\begin{array}{l}\text { Interpretar } \\
\text { Representar }\end{array}$ & $\begin{array}{l}\text { Evaluación } \\
\text { permanente de avance }\end{array}$ \\
\hline & Apoyos didácticos & $\begin{array}{l}\text { Para comprensión } \\
\text { Para interpretación }\end{array}$ & $\begin{array}{l}\text { Representar } \\
\text { interpretar } \\
\text { Identificar }\end{array}$ & $\begin{array}{l}\text { Evaluación } \\
\text { permanente de avance }\end{array}$ \\
\hline & $\begin{array}{l}\text { Percepción } \\
\text { práctica }\end{array}$ & $\begin{array}{l}\text { Ejemplificación } \\
\text { Fijación del } \\
\text { concepto }\end{array}$ & $\begin{array}{l}\text { Graficar } \\
\text { Comparar } \\
\text { Interpretar } \\
\text { Representar } \\
\text { identificar (todas) }\end{array}$ & $\begin{array}{l}\text { Evaluación } \\
\text { permanente de avance }\end{array}$ \\
\hline \multirow[t]{3}{*}{$\begin{array}{l}2 \text { Aplicación } \\
\text { práctica } \\
\text { (proceso) }\end{array}$} & $\begin{array}{l}\text { Lógica del } \\
\text { procedimiento del } \\
\text { cálculo de límites }\end{array}$ & $\begin{array}{l}\text { Objetivo } \\
\text { Tipos de límites } \\
\text { Procedimientos y su } \\
\text { lógica } \\
\text { Cálculo } \\
\text { propiamente tal }\end{array}$ & $\begin{array}{l}\text { Todas las } \\
\text { anteriores y } \\
\text { Calcular, } \\
\text { Fundamentar }\end{array}$ & Evaluación individual \\
\hline & $\begin{array}{l}\text { Ejercitación del } \\
\text { cálculo de límites }\end{array}$ & $\begin{array}{l}\text { Taller práctico } \\
\text { resuelto por los } \\
\text { estudiantes en } \\
\text { equipos. } \\
\text { Esquema de pasos } \\
\text { Nomenclatura } \\
\text { Acuerdos } \\
\text { Representación } \\
\text { gráfica de la } \\
\text { respuesta }\end{array}$ & $\begin{array}{l}\text { Calcular } \\
\text { Fundamentar }\end{array}$ & Evaluación individual \\
\hline & $\begin{array}{l}\text { Aplicaciones a la } \\
\text { vida real }\end{array}$ & $\begin{array}{l}\text { Modelación } \\
\text { Aplicación } \\
\text { Resolver }\end{array}$ & $\begin{array}{l}\text { Interpretar } \\
\text { Representar } \\
\text { Calcular } \\
\text { Fundamentar } \\
\text { Modelar }\end{array}$ & Evaluación individual \\
\hline
\end{tabular}




\begin{tabular}{lll}
\hline 3 Desarrollo Crítico & En Capacidad de Resolver & Evaluación oral \\
( salida) & 1. Ejercicios Conceptuales & \\
& 2. Ejercicios de Cálculo \\
& 3. Ejercicios de Razonar y Argumentar
\end{tabular}

\section{Fuente: Elaboración propia.}

\section{Conclusiones}

Muchos años se ha tratado acerca de las dificultades en el aula de matemáticas y en diferentes contextos educativos y el problema es bastante complejo debido a que la enseñanza de la matemática y en realidad todo tipo de enseñanza numérica, maneja muchos aspectos y muchas variables a considerar que afectan directamente al proceso de enseñanza y aprendizaje. En este artículo no se mencionaron algunas, por ejemplo: el sistema de creencias de los estudiantes y profesores; las nuevas metodologías participativas; las cambiantes generaciones de estudiantes influenciados por las Tics, incluso hasta elementos ambientales la disposición del aula de clases. Sin embargo se debe atacar el problema en forma moderada interviniendo en los elementos más manejables y más representativos para el aprendizaje.

Reconocemos que los sistemas que se pueden proponer, como este que estamos presentando, puede ser muy útil y no se debe esperar considerar que el aprendizaje estará garantizado y que sea la solución al problema, sino que, debemos considerarlo como un aporte al proceso de enseñanza y aprendizaje, que se enmarca como una mejora o un camino para encontrar un sistema mejor que pueda obtener mejores resultados.

Este sistema básico de formación de habilidades nace luego de la aplicación del modelo de Ramírez en los años 1998-1999 y como resultado de la experiencia en algunos años introduciendo adaptaciones cada vez, tratando de encuadrar mejoras y si efectivamente en muchos casos, ha dado buenos frutos, pero todavía se necesita seguir conjugando tal vez, con mejores metodologías y nuevos elementos.

En el sistema propuesto sólo se llega hasta la tercera fase del modelo de CórdovaOliveros, puesto que para avanzar a las etapas de Resolución de problemas e Innovación se necesita mucho más tiempo de desarrollo.

\section{Bibliografía}

Bermúdez, R., \& Rodríguez, M. (1996). Teoría y metodología del aprendizaje. La Habana.

Cabrera, B., \& Mazzarella, C. (2001). Vygotsky: enfoque socio-cultural. Educere, 41-44.

Córdova, N., \& Estay-Niculcar, C. (2002). Elementos de innovación docente y su impacto en la mejora del aprendizaje: modelo de congruencia y su aplicación en la mejora de las matemáticas en Guayaquil. . CIDUI 2002, Congreso Internacional "Docencia Universitaria". Tarragona: Actas Del Cidui. 
Córdova, N., \& Oliveros, E. (2014). La Matemática Superior y las Competencias. Gaceta sansana, 55-66.

Delgado, J. (1999). La enseñanza de la resolución de problemas matemáticos. la Habana: Base de datos AGIC-CREA.

Ferrer, M. (2000). La resolución de problemas en la estructuración de un sistema de habilidades en la escuela media cubana. Matemáticas. La Habana.

Ramírez P., C., Recabaren, M., \& Palma, A. (1988). Manual de Capacitación pedagógica. Valparaíso: Dirección de Instrucción de la Armada. Chile.

Rico, L. (1997). Los organizadores del currículo de matemáticas. La educación matemática en la enseñanza secundaria, 39-60.

Santaolalla, E. (2009). Matemáticas y estilos de aprendizaje. Estilos de Aprendizaje, nº4, Vol. 2, 56-69. 